\title{
A hybrid model of self organizing maps and least square support vector machine for river flow forecasting
}

\author{
S. Ismail ${ }^{1}$, A. Shabri ${ }^{2}$, and R. Samsudin ${ }^{3}$ \\ ${ }^{1}$ Department of Mathematics, Science Faculty, Universiti Teknologi Malaysia, Malaysia \\ ${ }^{2}$ Department of Software Engineering, Faculty of Computer Science and Information System, \\ Universiti Teknologi Malaysia, Malaysia
}

Correspondence to: S. Ismail (ismail.shuhaida@gmail.com), A. Shabri (ani@utm.my)

Received: 18 May 2010 - Published in Hydrol. Earth Syst. Sci. Discuss.: 18 October 2010

Revised: 31 August 2012 - Accepted: 11 September 2012 - Published: 26 November 2012

\begin{abstract}
Successful river flow forecasting is a major goal and an essential procedure that is necessary in water resource planning and management. There are many forecasting techniques used for river flow forecasting. This study proposed a hybrid model based on a combination of two methods: Self Organizing Map (SOM) and Least Squares Support Vector Machine (LSSVM) model, referred to as the SOM-LSSVM model for river flow forecasting. The hybrid model uses the SOM algorithm to cluster the entire dataset into several disjointed clusters, where the monthly river flows data with similar input pattern are grouped together from a high dimensional input space onto a low dimensional output layer. By doing this, the data with similar input patterns will be mapped to neighbouring neurons in the SOM's output layer. After the dataset has been decomposed into several disjointed clusters, an individual LSSVM is applied to forecast the river flow. The feasibility of this proposed model is evaluated with respect to the actual river flow data from the Bernam River located in Selangor, Malaysia. The performance of the SOM-LSSVM was compared with other single models such as ARIMA, ANN and LSSVM. The performance of these models was then evaluated using various performance indicators. The experimental results show that the SOM-LSSVM model outperforms the other models and performs better than ANN, LSSVM as well as ARIMA for river flow forecasting. It also indicates that the proposed model can forecast more precisely, and provides a promising alternative technique for river flow forecasting.
\end{abstract}

\section{Introduction}

Hydrological data such as flows and rainfall are the basic information used in the design of water resource systems. Knowledge about the characteristics and volume of river flow is very important, especially for predicting the future river flow in the monsoon season where the heavy rainfall may cause heavy river flow, potentially causing problems such as flooding and erosion. Reduced river flow is likely to restrict the supply of water for domestic use and industrial and hydroelectric power generation. Therefore, the ability to forecast future river flow would be beneficial in the field of water management and help in the design of flood protection works in urban areas and for agricultural land.

In hydrology, different types of models are used such as lumped conceptual models, physically-based models, also known as knowledge-driven modelling, and empirical models, also known as data-driven modelling. By using knowledge driven modelling, the other catchment variables such as catchment characteristics (size, shape, slope and storage characteristics of the catchment), and geomorphologic characteristics of a catchment (topography, land use patterns, vegetation and soil types that affect the infiltration) must be considered because it is hypothesized that forecasts could be improved if catchment characteristic variables which affect flow were to be included (Jain and Kumar, 2007; Dibike and Solomatine, 2001).

River flow forecast can be generated using two types of mathematical models: rainfall-runoff models and river flow models. The rainfall-runoff models use both climatic and hydrologic data, while river flow models only use the 
hydrologic data. The river flow process in a catchment area is a complex process that can be affected by many inter related physical factors. The factors affecting the river flow response of a catchment subjected to rainfall input include storm characteristics, such as intensity and duration of rainfall events, and so on. Moreover, the conceptual rainfallrunoff models need a large amount of data for calibration and validation purposes and are usually computationally expensive and very time consuming (Grayson et al., 1992; Jain and Kumar, 2007). Although combining other variables may improve their prediction accuracy, but in practice, especially in developing countries like Malaysia, such information is often either not available or difficult to obtain. Moreover, the influence of these variables and many of their combinations in generating river flow is an extremely complex physical process, especially due to the data collection of multiple inputs and parameters, which vary in space and time and which are not clearly understood (Zhang and Govindaraju, 2000; Jain and Kumar, 2007).

Owing to the complexity of this process, many researchers are focusing on river flow forecasting, which only considers the past river flow data, because it offers a rapid development and minimum information requirement (Adamowski and Sun, 2010; Kisi, 2004, 2008; Wang et al., 2009). This data-driven modelling by using historical data which are based on extracting and re-using the information that is implicitly contained in the hydrological data without taking directly into account any physical load that underlies the rainfall-runoff process provides accurate and rapid development time with minimum data information.

River flow forecasting is an important yet difficult task in the field of hydrology because predicting future events involves a decision-making process where the ability to predict future river flow will provide the right edge and assist the engineers in terms of flood control management, and provide some benefits in the areas of water supply management (Viessman et al., 1989). Accurate continuous data collections on the catchment area are needed to produce a good river flow forecast. There are many forecasting techniques that have been proposed in the literature for river flow forecasting. The most comprehensive of all popular and widely-known statistical methods used in time series forecasting is the Autoregressive Integrated Moving Average (ARIMA) model, also known as the Box Jenkins model. Several studies have shown that ARIMA can be trusted as a reliable model in water resources time series analysis (Muhamad and Hassan, 2005; Huang et al., 2004; Modarres, 2007; Fernandez and Vega, 2009; Wang et al., 2009) ).

Among the non-linear approaches, the Artificial Neural Network (ANN) is the most widely used for time series forecasting and has been successfully employed in the modelling of a wide range of hydrologic contexts (Maier and Dandy, 2000; Dibike and Solomatine, 2001; Bowden et al., 2005; Dolling and Varas, 2003; Muhamad and Hassan, 2005; Kisi, 2008; Wang et al., 2009; Keskin and Taylan, 2009; Luk et al., 2000; Hung et al., 2009; Affandi and Watanabe, 2007; Birkinshaw et al., 2008; Corzo et al., 2009). ANN provides an attractive alternative tool for forecasting and has shown its nonlinear modelling capabilities in data time series forecasting. However, the selection of an optimal network structure (layers and nodes) and training algorithms always needs the attention of modellers (Maier and Dandy, 2000). The network structure is usually determined by using a trial-anderror approach (Kisi, 2004).

Recently, the support vector machine (SVM) method, which was suggested by Vapnik (1995), has been used in hydrological modelling such as stream flow forecasting (Asefa, et al., 2006), flood stage forecasting (Yu et al., 2006), rainfall runoff modelling (Dibike et al., 2001; Elshorbagy et al., 2010a, b), etc. However, the standard SVM is based on the structural risk minimal principal and involves complicated quadratic programming methods, which are often time consuming and have a higher computational burden because of the required constrained optimization programming.

As a simplification of SVM, Suykens et al. (2002) proposed the use of the least squares support vector machines (LSSVM). LSSVM has been used successfully in various areas of pattern recognition and regression problems (Hanbay, 2009; Kang et al., 2008). LSSVM encompasses similar advantages to SVM, but its additional advantage is that it only requires the solving of a set of linear equations, which is much easier and computationally simpler. The method uses equality constraints instead of inequality constraints and adopts the least squares linear system as its loss function, which is computationally attractive. LSSVM also has good convergence and high precision. Hence, this method is easier to use than quadratic programming solvers in the SVM method. Extensive empirical studies (Wang and $\mathrm{Hu}, 2005$ ) have shown that LSSVM is comparable to SVM in terms of general performance. In the area of water resources, the LSSVM method has received very little attention in the literature and there are only a few applications of LSSVM in the modelling of environmental and ecological systems such as water quality prediction (Yunrong and Liangzhong, 2009).

Clustering analysis, which is the subject of active research in several fields such as statistics, pattern recognition, machine learning, and data mining, is to partition a given set of data or objects into clusters or groups or classes. It also has been applied in a large variety of applications, for example character recognition, document retrieval, etc. The goal of clustering analysis is to group similar objects together. Cluster analysis is a standard method of statistical multivariate analysis, and it can reduce large and complex datasets to a small number of data groups where members of the group share similar characteristics (Lin et al., 2006). The clustering algorithms attempt to partition data into clusters or natural groups such that the data within a cluster are as similar as possible, and data belonging to different clusters are as dissimilar as possible. Therefore, SOM pursues a goal that is conceptually different from that of clustering (Wu and Chow, 
2004). However, SOM has been successfully utilized as a first step in clustering algorithms.

The Self Organizing Map (SOM), proposed by Kohonen (2001), is one category of ANN that was first used as an information-processing tool in the fields of speech and image recognition. SOM has been successfully applied in clustering, classification, estimation, prediction and data mining (Vesanto and Alhoniemi, 2000; Kohonen, 2001). SOM can be used as a clustering tool since it converts the non linear statistical relationship between high dimensional data into simple geometric relationships of their image points on a low-dimensional display. From that, the data points which show similar properties are placed close to each other within the output of the SOM algorithm (Budayan et al., 2009). After the SOM training is finished, one can figure out the number of clusters directly by eye according to the two-dimensional map (Lin and Chen, 2005). Clustering the dataset may seem unnecessary; however, it is an important task especially when dividing a complex problem into several smaller and simpler problems can more easily be solved compared with the original problem (Jacobs et al., 1991; Lin and Chen, 2006; Lin and Wu, 2007; Wu and Chau, 2009; Wu et al, 2009).

SOM has attracted increasing interest for water resources application, such as the classification of satellite imagery data and rainfall estimation (Murao et al., 1993), rainfallrounoff modelling (Hsu et al., 2002), typhoon-rainfall forecasting (Lin and Wu, 2009), river flood forecasting (Chang et al., 2007), water resource problems (Kalteh et al., 2008), and model evaluation (Herbst and Casper, 2008; Herbst et al., 2009). The advantages of SOM compared with the other clustering methods have been extensively discussed in the literature (Chen et al., 1995; Mangiameli et al., 1996; Lin and Chen, 2006). Mangiameli et al. (1996) showed that the SOM performed the best when compared to seven other hierarchical clustering methods. Lin and Chen (2006) recommend SOM as an alternative to the identification of homogeneous regions for regional frequency analysis where the results showed that the SOM determines the cluster membership more accurately than the K-means method and Ward's method. In addition, the SOM is more robust than the traditional clustering methods. Lin and Chen (2005) apply the SOM clustering for predicting the groundwater head at HsiuLin Station, Taiwan. Among 240 datasets, the first 192 samples are used for training and the remaining 48 samples are used for testing. The entire datasets are then mapped into $14 \times 14$ map sizes. The map is then divided into 15 regions or clusters to continue with the prediction model using RBFN.

Improving forecasting accuracy, especially in time series forecasting, is an important yet often difficult task facing decision-makers in many areas. Using hybrid models has become common practice to improve forecasting accuracy. There are several studies that show that hybrid models can be an effective way to improved the accuracy of forecasting, compared to using single models separately (Zhang, 2003;
Jain and Kumar, 2007; Chen and Wang, 2007; Pai and Lin, 2005; Hsu et al., 2009). For instance, Lin and Wu (2009) proposed a combination of SOM and MLP in order to forecast the typhoon rainfall at Tanshui River Basin. SOM was used to analyze and divide the input data into distinct clusters. The second step involved an individual relationship between the input and output data constructed by a specific MLP. For evaluating the forecasting performance of the proposed model, an application was conducted. The results show that the proposed model can forecast more precisely than the model developed by the conventional neural network approach. Srinivas et al. (2009) combined a SOM and fuzzy clustering for regional flood frequency analysis for watersheds data from Indiana, USA. Results show that the proposed approach performs better in estimating flood quantiles at ungauged sites.

In recent years more hybrid models have been proposed, which combine a clustering technique with local forecasting models that are more accurate since these models are more specialised and have successfully solved many predictions problems, such as a combination of SOM with ANN (Pal et al., 2003; Lin and Wu, 2009; Wang and Yan, 2004), SOM with SVM (Cao, 2003; Fan and Chen, 2006; Fan et al., 2007; Huang and Tsai, 2009), SOM with Radial Basis Function (Lin and Chen, 2005), ANN with K-means (Corzo and Solomantine, 2007) and other models (Chang and Liao, 2006; Chang et al., 2007, 2008). Although the idea of these hybrid models is interesting and promising, it still need to be tested using a river flow time series.

Based on the same idea by Tay and Cao (2001) and Hsu et al. (2009), this study aims to explore the application of hybrid technique and to test the capability and effectiveness of the idea of hybrid modelling which combines the SOM with the LSSVM (SOM-LSSVM). The hybrid model SOMLSSVM is then proposed for river flow forecasting in order to improve the accuracy of prediction. With the advantages of the data analysis technique developed by SOM and the capability of LSSVM, the proposed hybrid model is expected to be useful for river flow forecasting. The results of the predictions by the SOM-LSSVM model are compared with a forecasting model developed by conventional ARIMA, ANN and LSSVM models. To verify the application of this approach, the monthly river flow for Bernam River located in Selangor, Malaysia was analyzed as a case study in this research.

\section{Data-driven modelling}

Data-driven modelling is considered as a tool in building a model that will replace the knowledge-driven modelling in describing a physical behaviour (Solomatine et al., 2008). However, the data-driven models may not correctly represent the input-output mapping if the modelled system changed during the time when the data are collected. Therefore, it is necessary for the entire dataset to go through a statistical 
analysis test to prove whether there are some other trends in the dataset. Trend analysis on time series data has been proven to be a useful tool for effective water resources planning, design, and management (Douglas et al., 2000; Hamilton et al., 2001), since trend detection of hydrological variables such as river flow provide useful information on the possibility of change tendency of the variables in the future (Yue and Wang, 2004).

Another issue that still remains a question is the number of the appropriate data that can be used to predict future river flows by using data-driven modelling. Number of data also plays an important role in predicting future river flows. For example, Kisi and Cimen (2010) used the monthly river flow data of Canakdere River and Goksudere River in their research. The observed data are $40 \mathrm{yr}$ (480 months) along with an observation period between 1960 and 1999 for both stations. Jain and Kumar (2007) applied their proposed model using monthly river flow data for a period of $62 \mathrm{yr}$ (1911-1972) derived from the Colorado River at Lees Ferry, CO, USA, for modelling a hydrologic time series forecasting. Usually, the monthly data are used to estimate the water demand and the water supply (Srikanthan and McMahon, 2001). Although river flow forecasting models using historical river flow time series data may lack the ability to provide physical interpretation and insight into catchment processes, they are nevertheless able to provide relatively accurate flow forecasts and become more and more popular in hydrological modelling due to their rapid development times and minimum information requirements.

\section{Forecasting models}

This section presents the ARIMA, ANN, LSSVM and SOMLSSVM models used for river flow forecasting. The choice of these models in this study was due to the fact that these methods have been widely and successfully used in time series forecasting.

\subsection{The Autoregressive Integrated Moving Average Model}

The Box-Jenkins model, also know as Autoregressive Integrated Moving Average (ARIMA), was introduced by Box and Jenkins (1970) and has been one of the most popular approaches in the area of forecasting. The order of an ARIMA model is represented by ARIMA $(p, d, q)$ and the order of the seasonal ARIMA or SARIMA model is represented by ARIMA $(p, d, q) \times(P, D, Q)_{\mathrm{s}}$, where the term $(p, d, q)$ is the order of the non-seasonal and $(P, D, Q)_{\mathrm{s}}$ is the order of the seasonal. The general ARIMA models are a compound of a seasonal and non-seasonal part and are represented in the following manner:

$\phi_{p}(B) \Phi_{P}\left(B^{s}\right)(1-B)^{d}\left(1-B^{s}\right)^{D} x_{t}=\theta_{q}(B) \Theta_{Q}\left(B^{s}\right) a_{t}$, where $\phi(B)$ and $\theta(B)$ are polynomials of order $p$ and $q$, respectively; $\Phi\left(B^{s}\right)$ and $\Theta\left(B^{s}\right)$ are polynomials in $B^{s}$ of degrees $P$ and $Q$, respectively; $p$ is the order of non-seasonal auto regression; $d$ is the number of regular differencing; $q$ is the order of the non-seasonal moving average; $P$ is the order of seasonal autoregression; $D$ is the number of seasonal differencing; $Q$ is the order of seasonal moving average; $B$ is the backward shift operator, and $s$ is the length of the season. Random errors $a_{t}$ are assumed to be independently and identically distributed with a mean of zero and a constant variance of $\sigma^{2}$.

The ARIMA model involves four steps, which are the identification step, estimation step, diagnostic checking step and forecasting step. In the identification step, the sample autocorrelation function (ACF) and sample partial autocorrelation function (PACF) are used to determine whether or not the series is stationary and if it is seasonal or non-seasonal. If the series is not stationary, then a transformation called differencing is needed until the series reaches the stationary level. ACF as well as PACF are used to choose a tentative model. Once a tentative model is identified, the parameters of the model are estimated. Diagnostic checking using the ACF and PACF of the residuals is carried out, basically to check whether the model's assumptions about the error $a_{t}$ are satisfied. If the model is not adequate, a new tentative model should be identified followed by the steps of parameter estimation and model verification. The process is repeated several times until finally a satisfactory model is selected. The forecasting model is then used to compute the fitted values and forecasts values.

\subsection{Artificial neural network}

The ANN is flexible computing, which has been extensively studied and used for time series forecasting in many areas of science and engineering since early 1990 . The most commonly used ANN in the field of water resources and hydrology is the feed forward multi layer perceptron (MLP), which consists of three layers: the first is the input layer where the data are introduced to the network, the second layer is the hidden layer where the data are processed, and the final layer is the output layer where the results of the given input are produced. The structure of a feed-forward ANN is shown in Fig. 1.

Mathematically, a three-layer MLP with $p$ input nodes, $q$ hidden nodes and one output node can be expressed as

$y_{t}=g\left(\sum_{j=1}^{q} w_{j} f\left(\sum_{i=1}^{p} w_{i} x_{t-i}\right)\right)$,

where $y_{t}$ is the output layer, $x_{t-i}$ is the input of the network, $w_{i}$ is the connection weights between the input and hidden layer nodes, $w_{i}$ is the connection weights between hidden and output layer nodes, and $g($.$) and f($.$) are activation func-$ tions. The most common $g($.$) as the sigmoid function and$ 


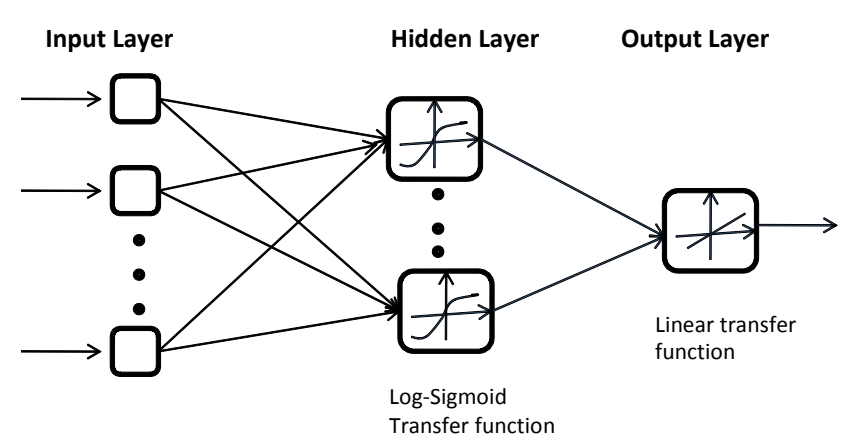

Fig. 1. The architecture Of three layers feed-forward backpropagation ANN.

$f($.$) as the linear function are adopted here. The equations$ for linear function and sigmoid function are as follows:

Linear: $\quad f(x)=\operatorname{purelin}(x)=x_{i}$

Log-Sigmoid: $\quad f(x)=\log \operatorname{sig}(x)=\frac{1}{1+\exp (-x)}$.

Training a network is an essential factor for the success of neural networks. Among the several learning algorithms available, backpropagation has been the most popular and most widely implemented learning algorithm for all neural network paradigms (Zou et al., 2007). In a backpropagation network, the weighted connections only feed activations in the forward direction from an input layer to the output layer. These interconnections are adjusted using an error convergence technique where the best match for the network's response is the desired response. Backpropagation is the most popular algorithm for training feed-forward MLP. For detailed reviews of ANN, along with their application in water resources and hydrology, the reader can be referred to Maier and Dandy (2000).

\subsection{Least Squares Support Vector Machine}

The Least Squares Support Vector Machine (LSSVM) is a modification of the standard Support Vector Machine (SVM), and was developed by Suykens and Vandewalle (Suykens, 2002). The basic LSSVM is used for the optimal control of non-linear Karush-Kuhn-Tucker systems for classification as well as regression. The LSSVM predictor is trained using a set of time series historic values as inputs and a single output as the target value. In the following, we briefly introduce LSSVM and its use in time series forecasting.

Consider a set of data $D=\left\{\left(x_{1}, y_{1}\right),\left(x_{2}, y_{2}\right), \ldots,\left(x_{n}, y_{n}\right)\right\}$, $x_{i} \in \Re^{p}, y_{i} \in \Re, x$ is the input vector, $y$ is the expected output and $n$ is the number of data. The LSSVM approximate the function in the following form:

$y(x)=w^{T} \varphi(x)+b$,

where $\phi(x)$ represents the high dimensional feature spaces, which is non-linearly mapped from the input space $x$. By combining the functional complexity and fitting error, the optimization problem of LSSVM is given as

min:

$J(w, \xi)=\frac{1}{2} w^{T} w+\frac{\gamma}{2} \sum_{i=1}^{n} \xi ;_{i}^{2}$

subject to:

$y(x)=w^{T} \varphi\left(x_{i}\right)+b+\xi_{i} i=1,2,3, \ldots, n$.

This formulation consists of equality instead of inequality constraints. To solve this optimization problem, the Lagrange function is constructed as

$$
\begin{aligned}
& L(w, b, \xi ; \alpha)=J(w, b, \xi) \\
& \quad-\sum_{i=1}^{n} \alpha_{i}\left\{w^{T} \varphi\left(x_{i}\right)+b-y_{i}+\xi_{i}\right\},
\end{aligned}
$$

where $\alpha_{i}$ are the Lagrange multipliers, which can be positive or negative. The solution of Eq. (8) can be obtained by partially differentiating with respect to $w, b, \quad \xi_{i}$ and $\alpha_{i}$

$$
\begin{cases}\frac{\partial L}{\partial w}=0 \rightarrow w=\sum_{i=1}^{n} \alpha_{i} \varphi\left(x_{i}\right) & \\ \frac{\partial L}{\partial b}=0 \rightarrow \sum_{i=1}^{n} \alpha_{i}=0 & \text { for } i=1,2,3, \ldots n . \\ \frac{\partial L}{\partial \xi_{i}}=0 \rightarrow \alpha_{i}=\gamma \xi_{i} \\ \frac{\partial L}{\partial \alpha_{i}}=0 \rightarrow w^{T} \varphi\left(x_{i}\right)+b-y_{i}+\xi_{i}=0\end{cases}
$$

After elimination of the variables $w$ and $\xi_{i}$, one obtains the following matrix solution:

$\left[\begin{array}{ll}0 & \mathbf{1}^{T} \\ \mathbf{1} \phi\left(x_{i}\right)^{T} \phi\left(x_{l}\right)+\gamma^{-1} I\end{array}\right]\left[\begin{array}{l}b \\ \alpha\end{array}\right]=\left[\begin{array}{l}0 \\ y\end{array}\right]$,

with $y=\left[y_{1}, y_{2}, \ldots, y_{l}\right], 1_{v}^{T}=[1,1, \ldots, 1], \alpha=\left[\alpha_{1}, \alpha_{2}, \ldots\right.$, $\left.\alpha_{l}\right]$. The kernel function can be defined as

$K\left(x_{i}, x_{j}\right)=\phi\left(x_{i}\right)^{T} \phi\left(x_{j}\right), \quad i, j=1,2, \ldots, n$.

This finally leads to the following LSSVM model for regression:

$y(x)=\sum_{i=1}^{n} \alpha_{i} K\left(x_{i}, x_{j}\right)+b$,

where $\alpha_{i}, b$ are the solutions to the linear system and $K\left(x_{i}, x_{j}\right)$ is a kernel function. The most popular kernel function is the Radial Basis Function (RBF), as shown in Eq. (13) (Liu and Wang, 2008; Gencoglu and Ulyar, 2009).

$$
K\left(x_{i}, x_{j}\right)=\exp \left(-\frac{\left\|x-x_{i}\right\|^{2}}{\delta^{2}}\right) .
$$




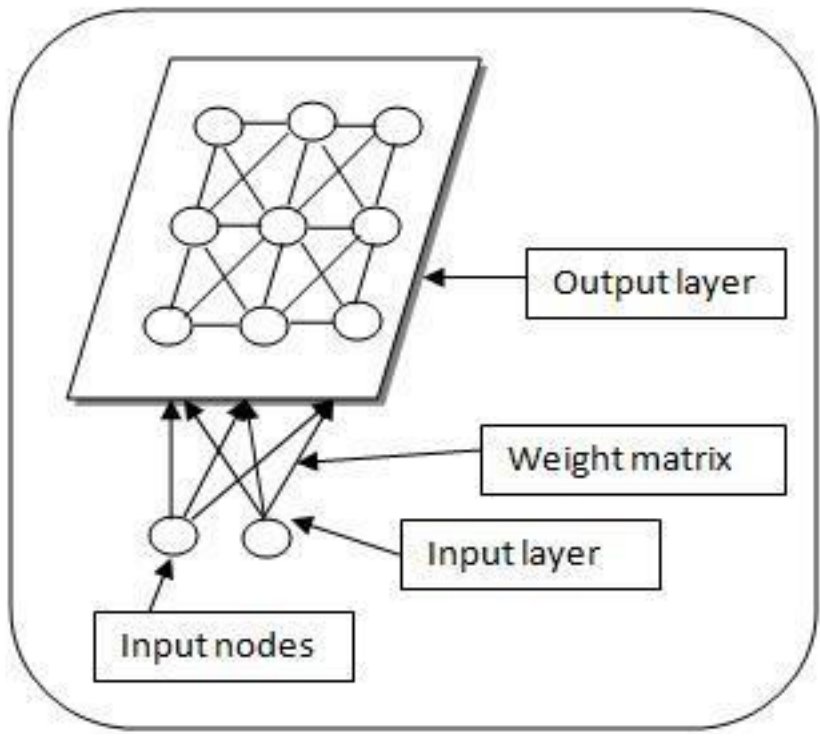

Fig. 2. The SOM architecture.

\subsection{Self organizing map}

SOM, also known as the Self Organizing Feature Map (SOFM), was proposed by Professor Teuvo Kohonen and therefore sometimes called the Kohonen Map (Kohonen, 2001), is an unsupervised and competitive learning algorithm. SOM has been used widely for data analysis in some areas such as economics, physics, chemistry as well as medical applications.

The objectives of SOM are to maximize the degree of similarity of patterns within a cluster, minimize the similarity of patterns belonging to different clusters, and then present the results in a lower-dimensional space. Basically, SOM consists of two layers of artificial neurons: the input layer, which accepts the external input signals; and the output layer, also called the output map, which is usually arranged in a twodimensional structure. Every input neuron is connected to every output neuron, and each connection has a weighting value attached to it. The architecture of SOM is shown in Fig. 2.

Output neurons will self organize to an ordered map, and neurons with similar weights are placed together. They are connected to adjacent neurons by a neighbourhood relation, dictating the topology of the map (Moreno et al., 2006). The concept of the learning algorithm for SOM is unsupervised and competitive. The training process of SOM is described below:

For simplicity, we assume that the input vector $\boldsymbol{X}$ of SOM is:

$\boldsymbol{X}=\left[x_{1}, x_{2}, \ldots, x_{n}\right]$,

where $n$ is the dimension of the input vector. The weight vector connecting the input vector to the hidden neuron $i$ is denoted by
$W_{i}=\left[w_{i 1}, w_{i 2}, \ldots, w_{i n}\right] \quad i=1,2, \ldots, m$.

The weights are initialised as small random numbers at the start of the training process. In competitive learning networks, the neurons compete among themselves to determine the winner by calculating the distance between the input vector and the weight vectors of all the neurons in the hidden layer. The winner $I$ is defined as the one whose weight vector is the closet to the input vector $X$ :

$I(X)=\min _{\forall i}\left\|X-W_{i}\right\| \quad i=1,2, \ldots, m$.

The Euclidean distance is often used as the similarity measure for SOM. The output neuron whose weight vector has the smallest distance from the input vector is called the winning neuron.

After determining the winning neuron, the lateral intersections between the winning neuron and its neighbourhood are calculated using the topological neighbourhood function. The neighbourhood function takes the form of a radial basis function that is appropriate for representing the biological lateral interaction (Kohonen, 2001; Rui Xu, 2009):

$h_{j i}(t)=\eta(t) \exp \left(\frac{-\left\|r_{j}-r_{i}\right\|^{2}}{2 \sigma^{2}(t)}\right)$,

where $\left\|r_{j}-r_{i}\right\|$ represents the Euclidean distance between the winning neuron $i$ and the neighbouring neuron $j$, and $\sigma(t)$ is the bandwidth of the radial basis kernel function.

Next, the weights of this winning neuron are adjusted according to the input patterns using the algorithm

$W_{i}(t+1)=W_{i}(t)+h_{j i}(t)\left(X-W_{i}(t)\right)$,

where $\eta(t)$ is the learning rate at time $t$ and $W_{i}(t+1)$ is the adjusted weight vector at time $(t+1)$.

After the weights have been updated, the winning neuron and the neighbourhood neurons become more similar to the corresponding input pattern. The process continues until convergence has been reached. Finally, the trained SOM is obtained.

\subsection{Integrating the SOM-LSSVM model}

Time series is a chronological sequence of observations of data points recorded sequentially in time. Time series forecasting is used to predict future values based on past values and other variables. However, the datasets are full with non-linearity. To address these issues, this study employs a hybrid model to better predict the future river flow. In this study, a hybrid model was implemented which combines the SOM clustering algorithm with the LSSVM model, as illustrated in Fig. 3. In the first stage, the datasets are divided into several groups or clusters. In order to do this, SOM is used to cluster the whole input space into regions where 


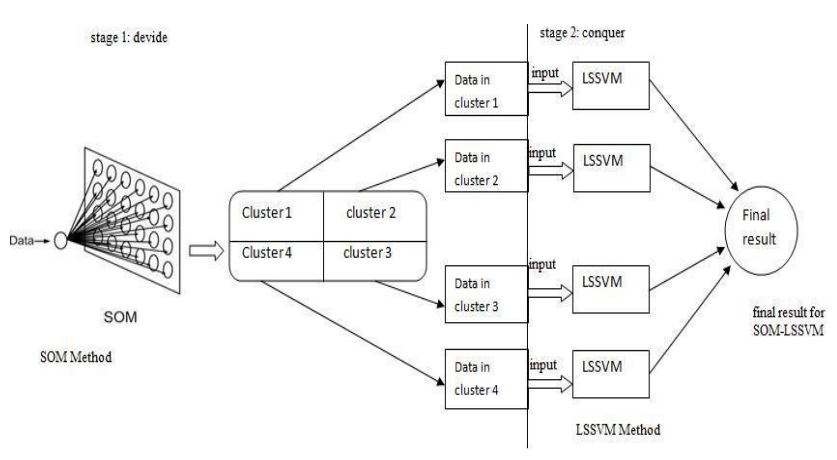

Fig. 3. The SOM-LSSVM architecture.

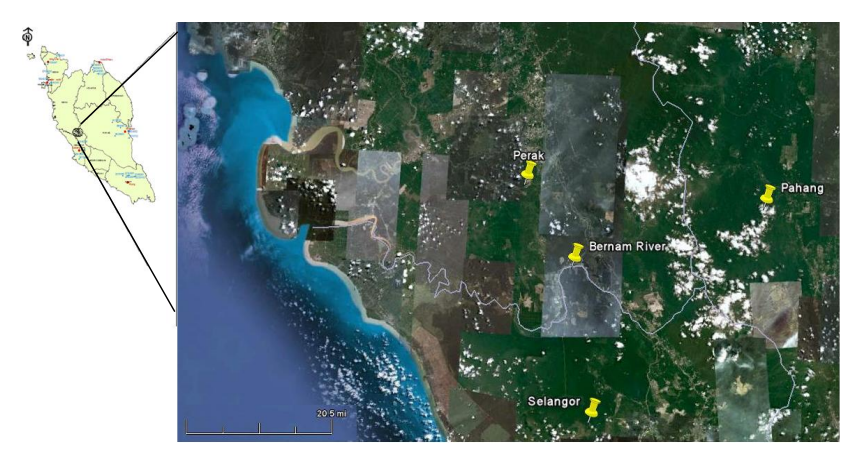

Fig. 4. The study area.

data points with similar statistical distribution are grouped together. Each group or cluster contains similar objects (Huang and Tsai, 2009). After the clustering of the data into several groups, LSSVMs are constructed for each cluster. LSSVM can conduct a better forecast for each group or cluster. As demonstrated by Tay and Cao (2001) and Hsu et al. (2009), this hybrid model can capture better results in the prediction of future river flow.

\section{The study area and data}

In this research, we examined the data obtained from the monthly river flow of the Bernam River located in Selangor, Penisular Malaysia. Bernam River is located between the states of Perak and Selangor, demarcating the border between the two states. The upper Bernam River basin has been identified as the ultimate and largest source of water supply from the Bernam River, especially for irrigation and the supply of drinking water. The study area is about $1090 \mathrm{~km}^{2}$ with a mean elevation of $19 \mathrm{~m}$, and the Bernam River monitoring station is the downstream outlet. The location of the Bernam River catchment is shown in Fig. 4.

The monthly river flow data of the Bernam River, consisting of 516 monthly records (January 1966 to December 2008), are used in this study. The data were first tested using Mann-Kendall test in order to detect any other trends in river flow data. After that, the dataset was then split up into two

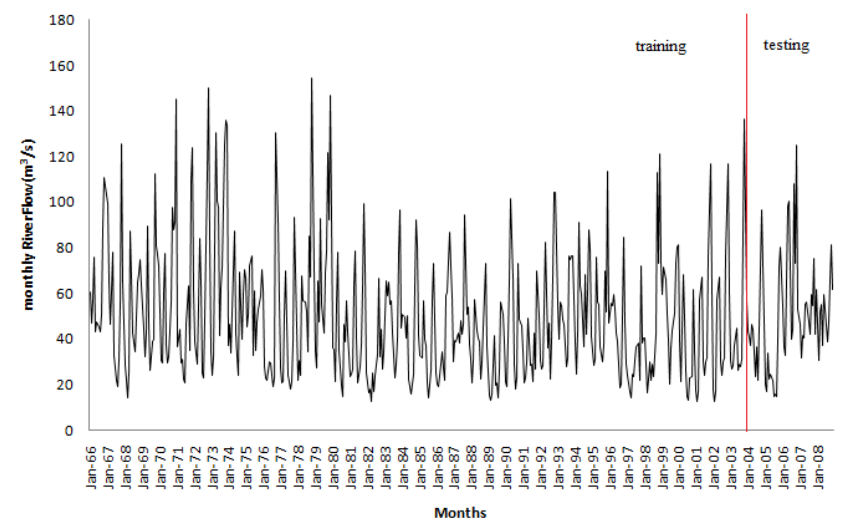

Fig. 5. Time series of monthly river flow of Bernam River (January 1966-Dezember 2008).

parts: training and testing, where the first dataset consisting of 456 monthly records (January 1966 to December 2003) was used for training, while the final dataset containing 60 mean monthly river flows (January 2004 to December 2008) was used for testing. Training data were used exclusively for model development and testing data were used to measure the performance of the model on untrained data. The testing set was also used to evaluate the forecasting ability of the model and to compare the proposed model with others. The recorded time series data for the Bernam River are shown in Fig. 5.

Solomatine et al. (2008) suggested that when splitting data into training and testing datasets, these sets should have similar distributions of low and high flow or similar properties of the input and output variables. However, it has been found that to generalise the training and testing sets with similar properties is not an easy task. Most studies suggest that the ratio of splitting for training and testing should be [70:30, 80:20, or 90:10]. The selection of the ratio could be based on the particular problem under consideration (Zhang et al., 1998; Firat, 2007; Kisi, 2008; Wang et al., 2009). Before the training process begins, data normalisation is often performed. The river flow was normalised in the range [0.1, 0.9] by the following equation:

$y_{t}=0.1+\frac{x_{t}}{1.2\left(x_{\max }\right)}$,

where $y_{t}$ represents the normalised data, while $x_{t}$ is the actual observation value and $x_{\max }$ represents the maximum value among the actual observation values.

\section{Input determination}

As with any data-driven model such as ANN and LSSVM, the selection of appropriate model inputs plays an extremely important role in their successful implementation since it provides the basic information about the system being modelled. In time series forecasting, usually insufficient attention 
Table 1. Model of input data.

\begin{tabular}{ll}
\hline Model & Input Data \\
\hline M1 & $y_{t}=f\left(x_{t-1}, x_{t-2}\right)$ \\
M2 & $y_{t}=f\left(x_{t-1}, x_{t-2}, x_{t-3}, x_{t-4}\right)$ \\
M3 & $y_{t}=f\left(x_{t-1}, x_{t-2}, x_{t-3}, x_{t-4}, x_{t-5}, x_{t-6}\right)$ \\
M4 & $y_{t}=f\left(x_{t-1}, x_{t-2}, x_{t-3}, x_{t-4}, x_{t-5}, x_{t-6}, x_{t-7}, x_{t-8}\right)$ \\
M5 & $y_{t}=f\left(x_{t-1}, x_{t-2}, x_{t-3}, x_{t-4}, x_{t-5}, x_{t-6}, x_{t-7}, x_{t-8}, x_{t-9}, x_{t-10}\right)$ \\
M6 & $y_{t}=f\left(x_{t-1}, x_{t-2}, x_{t-3}, x_{t-4}, x_{t-5}, x_{t-6}, x_{t-7}, x_{t-8}, x_{t-9}, x_{t-10}, x_{t-11}, x_{t-12}\right)$ \\
M7 & $y_{t}=f\left(x_{t-1}, x_{t-2}, x_{t-4}, x_{t-5}, x_{t-7}, x_{t-10}, x_{t-12}\right)($ Stepwise $)$ \\
M8 & $y_{t}=f\left(x_{t-1}, x_{t-2}, x_{t-12}, x_{t-13}, x_{t-14}, x_{t-24}, x_{t-25}, x_{t-26}, a_{t-12}, a_{t-24}\right)$ (ARIMA) \\
\hline
\end{tabular}

Table 2. The result for the training and testing using ANN model.

\begin{tabular}{|c|c|c|c|c|c|c|c|}
\hline \multirow{2}{*}{ Data } & \multirow{2}{*}{ Hidden Neurons } & \multicolumn{3}{|c|}{ Training } & \multicolumn{3}{|c|}{ Testing } \\
\hline & & MAE & RMSE & $R$ & MAE & RMSE & $R$ \\
\hline \multirow{4}{*}{ M1 (I =2) } & $\mathrm{I} / 2$ & 0.0967 & 0.1259 & 0.5377 & 0.0877 & 0.1096 & 0.5084 \\
\hline & I & 0.0961 & 0.1257 & 0.5392 & 0.0883 & 0.1105 & 0.4993 \\
\hline & $2 \mathrm{I}$ & 0.0969 & 0.1257 & 0.5398 & 0.0899 & 0.1113 & 0.4939 \\
\hline & $2 \mathrm{I}+1$ & 0.0971 & 0.1263 & 0.5333 & 0.0877 & 0.1110 & 0.4928 \\
\hline \multirow{4}{*}{ M2 (I =4) } & $\mathrm{I} / 2$ & 0.1150 & 0.1506 & 0.5426 & 0.1004 & 0.1316 & 0.5085 \\
\hline & I & 0.1135 & 0.1500 & 0.5478 & 0.1089 & 0.1372 & 0.4547 \\
\hline & $2 \mathrm{I}$ & 0.1135 & 0.1489 & 0.5571 & 0.1006 & 0.1295 & 0.5307 \\
\hline & $2 \mathrm{I}+1$ & 0.1126 & 0.1482 & 0.5628 & 0.1073 & 0.1339 & 0.4895 \\
\hline \multirow{4}{*}{ M3 (I =6) } & $\mathrm{I} / 2$ & 0.1098 & 0.1411 & 0.3363 & 0.0974 & 0.1200 & 0.3306 \\
\hline & I & 0.0940 & 0.1258 & 0.5426 & 0.0889 & 0.1126 & 0.4727 \\
\hline & $2 \mathrm{I}$ & 0.0909 & 0.1197 & 0.6013 & 0.0871 & 0.1087 & 0.5368 \\
\hline & $2 \mathrm{I}+1$ & 0.0936 & 0.1232 & 0.5684 & 0.0870 & 0.1112 & 0.4850 \\
\hline \multirow{4}{*}{ M4 (I=8) } & $\mathrm{I} / 2$ & 0.1125 & 0.1473 & 0.5756 & 0.1027 & 0.1255 & 0.5742 \\
\hline & I & 0.1100 & 0.1449 & 0.5937 & 0.0988 & 0.1284 & 0.5546 \\
\hline & $2 \mathrm{I}$ & 0.1075 & 0.1404 & 0.6263 & 0.1013 & 0.1237 & 0.5991 \\
\hline & $2 \mathrm{I}+1$ & 0.1067 & 0.1417 & 0.6176 & 0.1059 & 0.1321 & 0.5428 \\
\hline \multirow{4}{*}{ M5 (I =10) } & $\mathrm{I} / 2$ & 0.1245 & 0.1602 & 0.4584 & 0.1153 & 0.1466 & 0.3245 \\
\hline & I & 0.1025 & 0.1359 & 0.6565 & 0.101 & 0.1279 & 0.5749 \\
\hline & $2 \mathrm{I}$ & 0.1059 & 0.1402 & 0.6279 & 0.0955 & 0.1204 & 0.6284 \\
\hline & $2 \mathrm{I}+1$ & 0.1056 & 0.1396 & 0.6315 & 0.1121 & 0.1391 & 0.4912 \\
\hline \multirow{4}{*}{ M6 (I =12) } & $\mathrm{I} / 2$ & 0.0868 & 0.1143 & 0.6410 & 0.0750 & 0.1050 & 0.5738 \\
\hline & I & 0.0870 & 0.1154 & 0.6318 & 0.0812 & 0.1057 & 0.5632 \\
\hline & $2 \mathrm{I}$ & 0.0828 & 0.1105 & 0.6705 & 0.0873 & 0.1088 & 0.5543 \\
\hline & $2 \mathrm{I}+1$ & 0.0838 & 0.1107 & 0.6686 & 0.0790 & 0.1039 & 0.5780 \\
\hline \multirow{4}{*}{ M7 (I =7) } & $\mathrm{I} / 2$ & 0.0863 & 0.1150 & 0.6352 & 0.0755 & 0.1034 & 0.5863 \\
\hline & I & 0.0879 & 0.1155 & 0.6309 & 0.0775 & 0.1078 & 0.5447 \\
\hline & $2 \mathrm{I}$ & 0.0852 & 0.1134 & 0.6476 & 0.0791 & 0.1086 & 0.5397 \\
\hline & $2 \mathrm{I}+1$ & 0.0850 & 0.1127 & 0.6533 & 0.0766 & 0.1048 & 0.5736 \\
\hline \multirow{4}{*}{ M8 (I =10) } & $\mathrm{I} / 2$ & 0.0567 & 0.0749 & 0.908 & 0.0697 & 0.0968 & 0.8256 \\
\hline & I & 0.0595 & 0.0756 & 0.9063 & 0.0758 & 0.1010 & 0.7937 \\
\hline & $2 \mathrm{I}$ & 0.0553 & 0.0716 & 0.9165 & 0.0605 & 0.0837 & 0.8610 \\
\hline & $2 \mathrm{I}+1$ & 0.0653 & 0.0834 & 0.8846 & 0.0785 & 0.0997 & 0.7755 \\
\hline
\end{tabular}

The bold formated line represent the best result for ANN technique. 

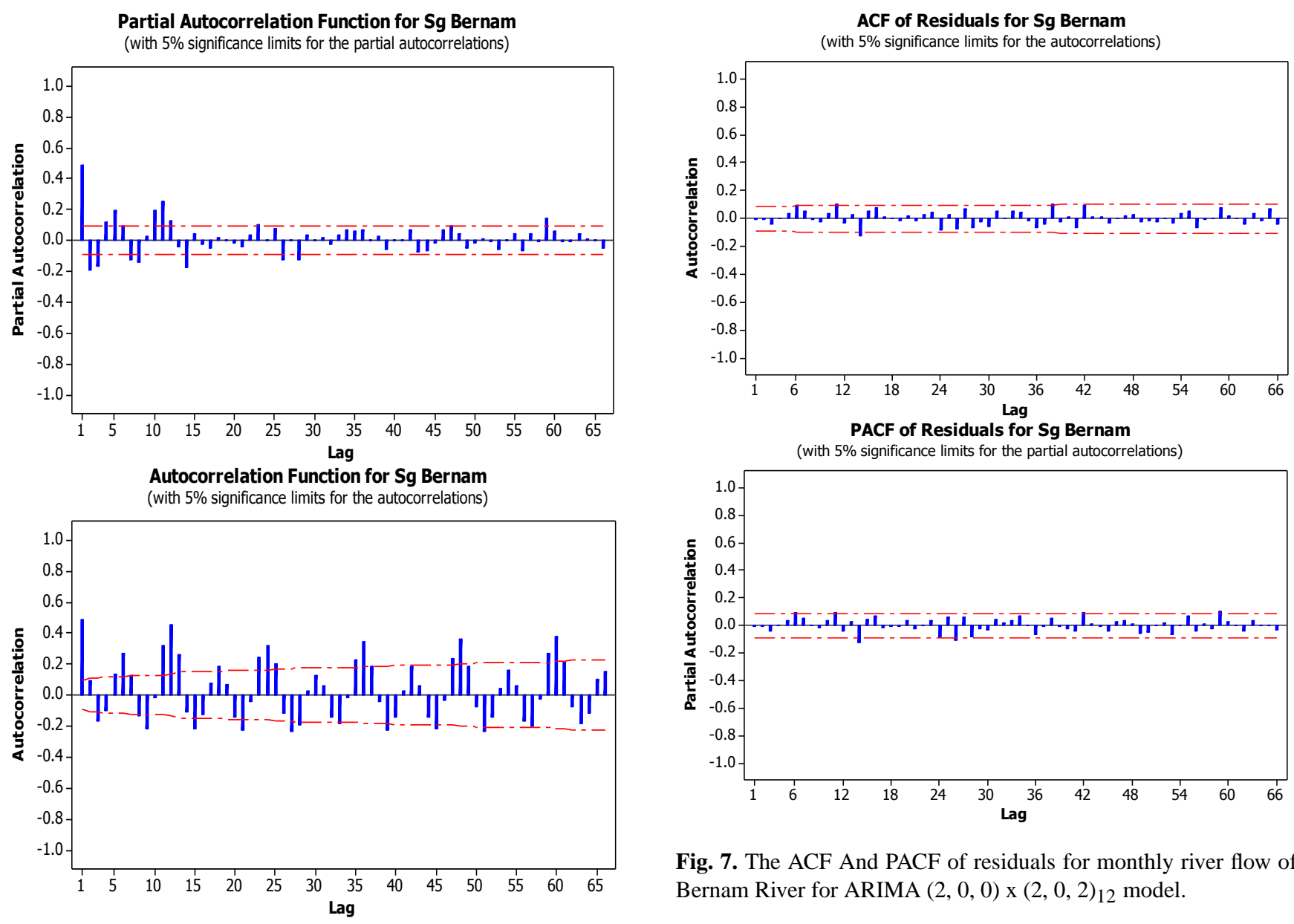

Fig. 7. The ACF And PACF of residuals for monthly river flow of Bernam River for ARIMA $(2,0,0)$ x $(2,0,2) 12$ model.

Fig. 6. ACF and PACF for monthly river flow of Bernam River.

is given to the task of selecting appropriate model inputs. Many papers reviewed failed to describe the input determination methodology used, and consequently raised doubts about the optimality of the output obtained (Bowden et al., 2005). Most researchers design experiments to help select the model inputs, while others adopt some empirical ideas. For example, Patil (1992) proposed model inputs based on 12 inputs for monthly data and four for quarterly data heuristically. Cheung et al. (1996) suggested maximum entropy principles to identify the time series lag structure. Tang and Fishwick claimed that the number of model inputs is simply the number of the autoregressive (AR) moving average components in the Box-Jenkins models. Refenes et al. (2003) suggested a stepwise method for determining the input for ANN models. Roadknight et al. (1997) used cross correlation analysis or principal component analysis as a guide for determining the input. Aqil et al. (2006) employed two statistical methods, i.e. autocorrelation (ACF) and partial autocorrelation $(\mathrm{PACF})$, to identify the appropriate input variables. Behzad et al. (2009) selected the best model inputs by trial and error according to minimum test errors in the ANN

and SVM modelling. Corzo et al. (2009) used correlation and average mutual information analysis involving different subbasin values using precipitation and river flow to determine the best input variables. Khashei and Bijari (2010) proposed an ARIMA model to determine the input variables in order to yield a more accurate forecasting model than ANN. The empirical results from three well-known real datasets showed that the proposed input variables can be an effective way to improve the forecasting accuracy achieved by ANN. The use of input variables from the data values of previous time series and the optimum number of input variables determined by trial and error has been reported by Firat (2007, 2008), Firat and Gungor (2007), Sivapragasam and Liong (2005), Juhos et al. (2008), Partal and Kisi (2007), among others.

Three approaches were used in this study to determine the models of input data. The first six approaches for the input data were chosen based on past river flow. The appropriate lags were chosen using a trial-and-error approach $\left(x_{t-1}, x_{t-2}, \ldots, x_{t-p}\right.$, where $p$ is $2,4, \ldots$, 12). It gives the number of inputs $(I)$ as $2,4,6,8$, 10, 12. The second and third approaches set the input vector nodes equal to the number of lagged variables from two statistical methods (i.e. stepwise multiple regression 
Table 3. The result for the training and testing using LSSVM model.

\begin{tabular}{lrrrrrr}
\hline \multirow{2}{*}{ Data } & \multicolumn{3}{c}{ Training } & & Testing \\
\cline { 2 - 7 } & MAE & RMSE & $R$ & MAE & RMSE & $R$ \\
\hline M1 & 0.0955 & 0.1248 & 0.5494 & 0.0858 & 0.1080 & 0.5191 \\
M2 & 0.0850 & 0.1120 & 0.6757 & 0.0860 & 0.1084 & 0.5207 \\
M3 & 0.0829 & 0.1120 & 0.6647 & 0.0797 & 0.1000 & 0.6092 \\
M4 & 0.0853 & 0.1134 & 0.6564 & 0.0812 & 0.1021 & 0.6037 \\
M5 & 0.0773 & 0.1035 & 0.7767 & 0.0800 & 0.1084 & 0.5232 \\
M6 & 0.0744 & 0.1018 & 0.7308 & 0.0744 & 0.0995 & 0.6191 \\
M7 & 0.0720 & 0.0970 & 0.7598 & 0.0771 & 0.1019 & 0.6756 \\
\hline M8 & 0.0486 & 0.0633 & 0.9259 & 0.0457 & 0.0611 & 0.8769 \\
\hline
\end{tabular}

analysis and the ARIMA model). Stepwise multiple regression analysis led to the selection of 7 input attributes $\left(x_{t-1}, x_{t-2}, x_{t-4}, x_{t-5}, x_{t-7}, x_{t-10}, x_{t-12}\right)$.

In ARIMA, the future value of a variable is assumed to be a linear function of several past observations and random errors (Zhang, 2003; Khashei and Bijari, 2010). In this study, ARIMA $(2,0,0) \times(2,0,2)_{12}$ is selected as the best model, as described in Sect. 6.1. Therefore, the functional form of the model input data using ARIMA is

$$
\begin{aligned}
& y_{t}=f\left(x_{t-1}, x_{t-2}, x_{t-12}, x_{t-13}, x_{t-14}, x_{t-24}, x_{t-25},\right. \\
& \left.x_{t-26}, a_{t-12}, a_{t-24}\right)
\end{aligned}
$$

where $y_{t}$ is the future value, $x_{t}$ is the past value at time $t$ and $a_{t}$ is the residual at time $t$, where ARIMA is used in order to generate the residuals.

\section{Evaluation of performance}

There are different types of performance evaluation that have been documented in the literature (Luchetta and Manetti, 2003; Goswami et al., 2005). The performance evaluation for each model should have at least a measure of absolute error, such as mean absolute error (MAE) or root mean square error (RMSE) (Legates and McCabe, 1999). Wang et al. (2006) stated that RMSE is a good performance evaluation measurement because it is very sensitive to even small errors, in which case it is better to compare the small differences in the model's performance.

The MAE and RMSE are defined as follows:

$$
\mathrm{MAE}=\frac{1}{n} \sum_{t=1}^{n}\left|y_{t}-\hat{o}_{t}\right|(20)
$$

$\mathrm{RMSE}=\sqrt{\frac{1}{n} \sum_{t=1}^{n}\left(y_{t}-\hat{o}_{t}\right)^{2}}$

where $y_{t}$ and $\hat{o}_{t}$ are the observed/actual and the predicted at the time $t$. The criteria to judge the best model are relatively small for MAE and RMSE in modelling and forecasting. Other than these, the correlation coefficient $(R)$ was also used as a performance measurement. $R$ was also used to test the ability of the model to capture the complex nature of the process that was being modelled (Jain and Kumar, 2007; Lin and $\mathrm{Wu}, 2009)$. It is a measure of how well the future outcomes are likely to be predicted by the model, where the predicted flows correlate with the observed flows. $R$ is defined as

$$
R=\frac{\frac{1}{n} \sum_{t=1}^{n}\left(y_{t}-\bar{y}\right)\left(\hat{o}_{t}-\overline{\hat{o}}\right)}{\sqrt{\frac{1}{n} \sum_{t=1}^{n}\left(y_{t}-\bar{y}\right)^{2}} \sqrt{\frac{1}{n} \sum_{t=1}^{n}\left(o_{t}-\overline{\hat{o}}\right)^{2}}},
$$

where $\bar{y}$ and $\overline{\hat{o}}$ are the mean observed and mean predicted river flow series, respectively, and $n$ is the number of data points. The $R$ value is used to evaluate the linear correlation between the observed and the predicted flow. Clearly, an $R$ value close to unity indicates a satisfactory result, while a low value or one close to zero implies an inadequate result.

\section{Experiment and results}

\subsection{Application of the ARIMA model}

Figure 5 shows the plots of the river flow time series, indicating that the time series are non-stationary and require a transformation. Samples of the autocorrelation function (ACF) and the partial autocorrelation function (PACF) for the series are plotted in Fig. 6. The ACFs curves for the monthly stream flow data decayed with mixture of sine wave pattern and exponential curves, which reflect the random periodicity of the data and indicate the need for seasonal MA terms in the model. In the PACF there were significant spikes present near lag 12 and 24, therefore indicating the series need for a seasonal AR process. The criteria used to judge the best model based on MSE show that ARIMA $(2,0,0) \times(2,0$, $2)_{12}$ is the best model. The model can be written as

$$
\begin{gathered}
\left(1-0.352 B-0.132 B^{2}\right)\left(1-0.603 B^{12}-0.395 B^{24}\right) \\
x_{t}=\left(1-0.477 B^{12}-0.460 B^{24}\right) a_{t},
\end{gathered}
$$

and can be rewritten as

$$
\begin{aligned}
& x_{t}=0.352 x_{t-1}+0.132 x_{t-2}+0.603 x_{t-12}-0.212 x_{t-13} \\
& +0.079 x_{t-14}+0.395 x_{t-24}-0.136 x_{t-25}-0.052 x_{t-26} \\
& -0.477 a_{t-12}-0.460 a_{t-24}+a_{t} .
\end{aligned}
$$

The ACF and PACF plots of the residuals of ARIMA $(2,0$, $0) \times(2,0,2)_{12}$ for the river flow series are shown in Fig. 7 . From the residual plot of the best ARIMA model, it was observed that the selected ARIMA $(2,0,0) \times(2,0,2)_{12}$ model passed the diagnostic checks and they were all white noise. For further analysis, we decided that the ARIMA model (2, $0,0) \times(2,0,2)_{12}$ is the best to use for comparison with the others. 
Table 4. The result for the training and testing using a hybrid model of SOM-LSSVM for different map sizes.

\begin{tabular}{|c|c|c|c|c|c|c|c|}
\hline \multirow{2}{*}{ Map Sizes } & \multirow{2}{*}{ Data } & \multicolumn{3}{|c|}{ Training } & \multicolumn{3}{|c|}{ Testing } \\
\hline & & MAE & RMSE & $R$ & MAE & RMSE & $R$ \\
\hline \multirow{8}{*}{$2 \times 2$} & M1 & 0.0680 & 0.0903 & 0.7964 & 0.0740 & 0.0872 & 0.7508 \\
\hline & M2 & 0.0655 & 0.0860 & 0.8205 & 0.0767 & 0.0963 & 0.6574 \\
\hline & M3 & 0.0758 & 0.1031 & 0.7259 & 0.0785 & 0.1020 & 0.6072 \\
\hline & M4 & 0.0686 & 0.0931 & 0.7925 & 0.0752 & 0.0975 & 0.6456 \\
\hline & M5 & 0.0770 & 0.1045 & 0.7250 & 0.0784 & 0.0988 & 0.6322 \\
\hline & M6 & 0.0869 & 0.1135 & 0.6495 & 0.0794 & 0.1022 & 0.5931 \\
\hline & M7 & 0.0758 & 0.1051 & 0.7126 & 0.0764 & 0.1011 & 0.6082 \\
\hline & M8 & 0.0212 & 0.0333 & 0.9782 & 0.0441 & 0.0620 & 0.8766 \\
\hline \multirow{8}{*}{$3 \times 3$} & M1 & 0.0747 & 0.0997 & 0.7445 & 0.0640 & 0.0860 & 0.7376 \\
\hline & M2 & 0.0532 & 0.0681 & 0.8908 & 0.0683 & 0.0879 & 0.7254 \\
\hline & M3 & 0.0760 & 0.1029 & 0.7271 & 0.0736 & 0.0917 & 0.7099 \\
\hline & M4 & 0.0736 & 0.0995 & 0.7517 & 0.0733 & 0.0908 & 0.7019 \\
\hline & M5 & 0.0721 & 0.1008 & 0.7504 & 0.0734 & 0.0951 & 0.6650 \\
\hline & M6 & 0.0685 & 0.0914 & 0.8049 & 0.0794 & 0.1067 & 0.5421 \\
\hline & M7 & 0.0735 & 0.0974 & 0.7599 & 0.0703 & 0.0971 & 0.6474 \\
\hline & M8 & 0.0278 & 0.0378 & 0.9705 & 0.0431 & 0.0622 & 0.8734 \\
\hline \multirow{8}{*}{$4 \times 4$} & M1 & 0.0537 & 0.0751 & 0.8640 & 0.0557 & 0.0691 & 0.8457 \\
\hline & M2 & 0.0561 & 0.0756 & 0.8642 & 0.0727 & 0.0884 & 0.7299 \\
\hline & M3 & 0.0649 & 0.0885 & 0.8105 & 0.0741 & 0.0937 & 0.6841 \\
\hline & M4 & 0.0696 & 0.0921 & 0.7947 & 0.0800 & 0.1010 & 0.6159 \\
\hline & M5 & 0.0647 & 0.0920 & 0.7990 & 0.0686 & 0.0916 & 0.7005 \\
\hline & M6 & 0.0645 & 0.0879 & 0.8253 & 0.0737 & 0.0992 & 0.6247 \\
\hline & M7 & 0.0701 & 0.0933 & 0.7830 & 0.0620 & 0.0894 & 0.7117 \\
\hline & M8 & 0.0348 & 0.0507 & 0.9485 & 0.0435 & 0.0647 & 0.8651 \\
\hline \multirow{8}{*}{$5 \times 5$} & M1 & 0.0659 & 0.0950 & 0.7715 & 0.0637 & 0.0876 & 0.7274 \\
\hline & M2 & 0.0446 & 0.0612 & 0.9127 & 0.0668 & 0.0838 & 0.7560 \\
\hline & M3 & 0.0646 & 0.0920 & 0.7911 & 0.0690 & 0.0865 & 0.7361 \\
\hline & M4 & 0.0550 & 0.0762 & 0.8762 & 0.0680 & 0.0870 & 0.7306 \\
\hline & M5 & 0.0555 & 0.0782 & 0.8646 & 0.0727 & 0.0931 & 0.6855 \\
\hline & M6 & 0.0385 & 0.0605 & 0.9231 & 0.0661 & 0.0850 & 0.7462 \\
\hline & M7 & 0.0761 & 0.0982 & 0.7848 & 0.0731 & 0.0969 & 0.6490 \\
\hline & M8 & 0.0401 & 0.0567 & 0.9299 & 0.0370 & 0.0492 & 0.9222 \\
\hline
\end{tabular}

Table 5. Comparative performance between ARIMA, ANN, LSSVM and SOM-LSSVM during the testing period.

\begin{tabular}{lrrr}
\hline Model & MAE & RMSE & $R$ \\
\hline ARIMA & 0.0767 & 0.1042 & 0.5842 \\
ANN & 0.0605 & 0.0837 & 0.8610 \\
LSSVM & 0.0457 & 0.0611 & 0.8769 \\
\hline SOM-LSSVM & 0.0370 & 0.0492 & 0.9222 \\
\hline
\end{tabular}

\subsection{Application of ANN model}

In this study, a typical three layer ANN model with a logsigmoid transfer function from the input layer to the hidden layer, and a linear function from the hidden layer to an output layer, are used for forecasting monthly river flow time series. The input and target data were normalised in the range $[0.1,0.9]$, because a sigmoid function was employed as the transfer function. The network was trained for 5000 epochs using the conjugate gradient descent backpropagation algorithm with a learning rate of 0.001 and a momentum coefficient of 0.9. The eight models of input data (M1-M8) with various numbers of input structures are trained and tested by ANN models, and the optimal number of neuron in the hidden layer was identified using several guidelines.

To help avoid the problem of over-fitting, some researchers have provided empirical rules to restrict the number of hidden nodes. In order to select an appropriate architecture, the following guidelines were used: " $I / 2$ " proposed by 
Kang (1991), "I" proposed by Tang and Fishwick (1993), " $2 I$ " proposed by Wong (1991), and " $2 I+1$ " proposed by Hecht-Nielsen (1990), where $I$ is the number of inputs.

It is common to use one test set for both validation and testing purposes, particularly with small datasets (Zhang, 2003). For ANN experiment, the dataset was split into two sections: training set and test set where the test set was used for validation and testing purposes, as demonstrated by Zhang (2003) and Kisi (2004). The networks that yielded the best results with the lowest MAE and RMSE and largest $R$ from the testing set were selected as the best ANN for the corresponding series. The effects of changing the number of hidden nodes on the RMSE, MAE and $R$ are shown in Table 2.

Table 2 shows the performance of ANN varying with the number of nodes in the hidden layer. For the training and testing period, the M8 model with 20 hidden nodes obtained the best results for MAE, RMSE and $R$, with statistics of 0.0553 , 0.0716 and 0.9163 , respectively. While in the testing phase, the M8 model with 20 hidden neurons was the best MAE, RMSE and $R$ with statistics of $0.0606,0.0837$ and 0.8610 , respectively. Hence, the ANN $(10,20,1)$ has been selected as the most appropriate ANN model for the Bernam River.

\subsection{Application of the LSSVM model}

There is no single proven theory that can be used to guide the selection of the number of inputs. In this study, the same input structures of the datasets M1 to M8 were used. The RBF was used as the kernel function for this study. In order to better evaluate the performance of the proposed approach, we considered a grid search of $\left(\gamma, \sigma^{2}\right)$ within ${ }_{n}$ the range 10 to 1000 , and $\sigma^{2}$ in the range 0.01 to 1.0 . For each hyper parameter pair $\left(\gamma, \sigma^{2}\right)$ in the search space, 5 -fold cross validation on the training set was performed to predict the prediction error. Table 3 shows the performance results obtained in the training and testing period of the LSSVM approach.

By considering these training and testing periods, the lowest MAE and RMSE and the largest $R$ for the series of data were calculated from the M8 model, resulting in statistics of $0.0486,0.0633,0.9259$ and $0.0457,0.0611$, 0.8769 , respectively.

\subsection{Application of the hybrid SOM-LSSVM model}

Determining the appropriate map sizes of clustering is very important for cluster validity and efficiency. For a SOM of large map sizes, input patterns will be grouped into a large number of clusters, which would cause each neuron to memorise one of the input patterns, although some clusters may only have one or two members. Such clustering results are not suitable for the forecasting analysis. On the other hand, if the map size is too small, then many different data groups might be lumped into the same category and the SOM will fail to show the topological relationships of the input patterns. Since there is no systematic or standard method for finding the optimal number of map sizes in the clustering algorithms, the optimal map size is obtained depending on the requirements of the user. In this paper, four map sizes are utilized, Kohonen of $2 \times 2,3 \times 3,4 \times 4$ and $5 \times 5$. When SOM is applied to perform cluster analysis, a SOM of a small dimension is the first choice. If the clustering result is reasonable and satisfactory, then the cluster analysis is accepted. Otherwise, a SOM of a larger dimension is chosen to analyse the input patterns, and this continues until a satisfactory result is obtained. In this study, only 4 clusters were considered to investigate the impacts of the number of map sizes on the performance. The same parameters were used as for the LSSVM's parameters for the single LSSVM model. Table 4 shows the predicted values of SOM-LSSVM for the various numbers of map sizes.

\subsection{Comparison}

In this section, the predictive capabilities of the proposed SOM-LSSVM model are compared with ARIMA, ANN and LSSVM for the Bernam monthly river flow. Furthermore, the MAE, RMSE, and $R$ are used to evaluate the performance of the ARIMA, ANN, LSSVM and SOM-LSSVM models. The statistical results of the different models are summarised in Table 5. From Table 5, it can be noted that the SOM-LSSVM model has the best performance with the lowest MAE and RMSE, and the largest $R$ for the testing phase. The single LSSVM is the second best model, followed by ANN. As can be seen in Tables 5, ARIMA has the worst performance based on MAE, RMSE and $R$.

In the testing phase, the SOM-LSSVM model improved the ARIMA model with about a $52.78 \%$ and $51.76 \%$ reduction in RMSE and MAE values, respectively, and with a $57.86 \%$ improvement of the forecast results for the $R$ value. SOM-LSSVM also produced some improvement over the ANN model with about a $45.16 \%$ and $38.84 \%$ reduction in RMSE and MAE, respectively, and some improvement in the forecast value of about $7.1 \%$ for $R$. As with LSSVM, the SOM-LSSVM model resulted in some improvement over LSSVM as well, with about a $19.47 \%$ reduction in RMSE, $19.03 \%$ reduction in MAE and improvement of $5.16 \%$ in the $R$ value.

Figure 8 shows the results obtained from the four models ARIMA, ANN, LSSVM and SOM-LSSVM compared with the actual river flow data for the last sixty months for the testing phase on the Bernam River data. From Fig. 8, all the models gave a close approximation to the actual observation data. It indicates that ARIMA, ANN, LSSVM and SOMLSSVM fit the monthly mean river flow better, and that these models are applicable for river flow forecasting. Meanwhile, Fig. 9 shows the scatter plots for the Bernam River, indicating that the estimates of the LSSVM and SOM-LSSVM models are closer to the actual river flow data than those of ARIMA and ANN. However, the value of $R$ and the fit line 

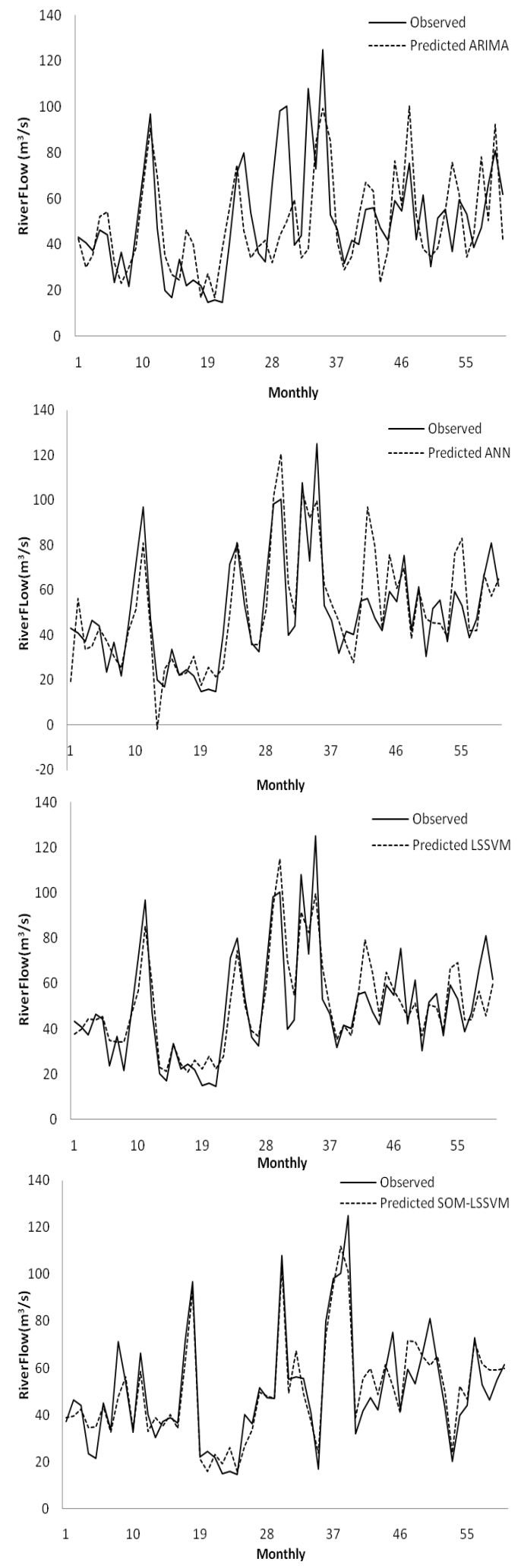

Fig. 8. Predicted and observed river flow during testing period by ARIMA, ANN, LSSVM and SOM-LSSVM for Bernam River.
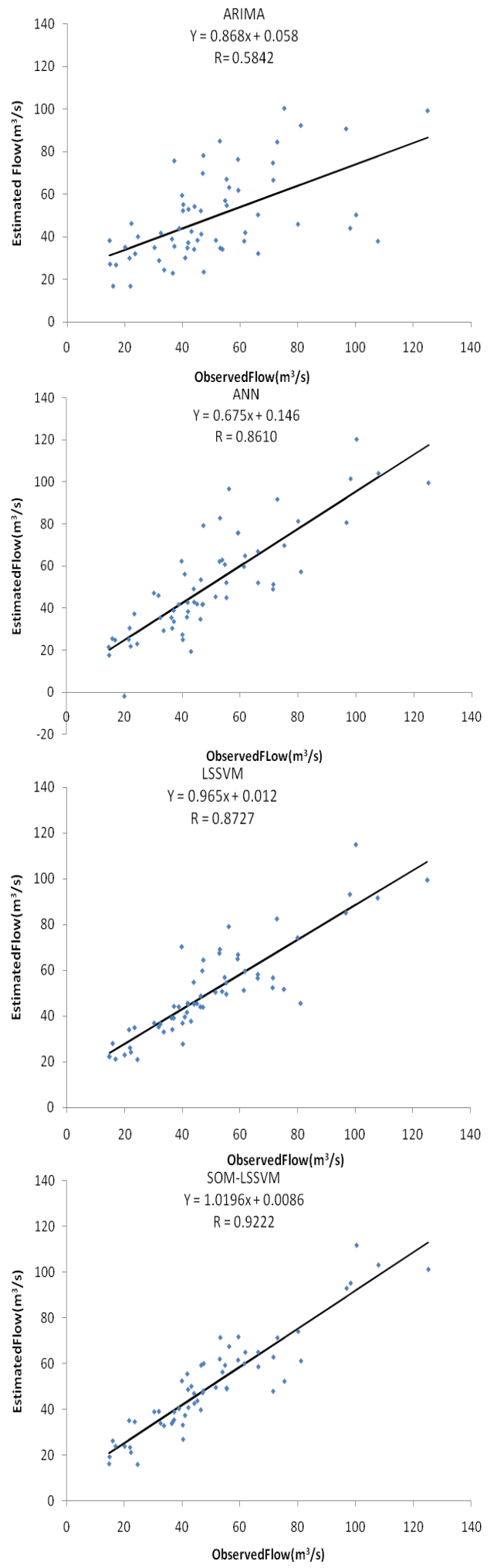

Fig. 9. The scatter plot of predicted and observed river flow during testing period using ARIMA, ANN, LSSVM and SOM-LSSVM for Bernam River. 
equation coefficients of the SOM-LSSVM are superior compared to the other models. The results indicate that the best performance can be obtained by the SOM-LSSVM model, followed by the LSSVM, ANN and ARIMA models. SOMLSSVM can also give a better prediction performance than ARIMA, ANN and LSSVM time series approaches. The results obtained in this study indicate that the SOM-LSSVM model is a powerful tool, as well as an alternative method for modelling river flow time series.

\section{Conclusions}

To improve the performance of river flow forecasting, a hybrid model based on a combination of SOM and LSSVM was proposed to predict monthly river flows. Before applying these models, the selections of the input data variables were conducted to determine the capability and suitability of the models to predict river flows. By using an evaluation on the performance test, the input data variables based on the ARIMA model were chosen as the optimal input factors. Next, SOM clustering technique was used to analyze these input data variables. The SOM algorithm clustered the entire input data into several disjointed clusters and after decomposing the data, LSSVM was used to predict the river flow. The result shows that the performance of river flow forecasting can be significantly enhanced by using the proposed hybrid SOM-LSSVM model.

To illustrate the capability of the SOM-LSSVM model, monthly river flow data from Bernam River were analyze in this study. The river flow data were varied per the number of input data used in the experiments. The number of input data variables were determine using the three approaches of past observation, stepwise regression analysis and ARIMA model. The experimental results show that SOM-LSSVM performs better than other models such as ARIMA, ANN and LSSVM. Through the comparison of four different models applied in monthly river flow forecasting, it can be concluded that SOM-LSSVM provides a promising alternative technique for river flow time series forecasting. It can also be concluded that the selections of input data variables also play an important role in the prediction as well as the number of Kohonen map sizes. In this study, only river flows data are considered for analysis, so future research can further test the idea of the hybrid model by employing the rainfall-runoff data, and using other clustering techniques such as K-Means or Fuzzy C-Means. Since this is an exploratory analysis of the hybrid SOM-LSSVM, the model should be further tested with another type of data with a variety of sample sizes to test the feasibility and ability of the SOM-LSSVM model. The idea of the hybrid model can also be tested on other time series data such as rainfall forecast, weather forecast, economic and so on to prove its capability and usability.
Acknowledgements. This research is supported by Zamalah Scholarship, Universiti Teknologi Malaysia and in part of E-Science, Ministry of Science, Technology and Innovation (MOSTI) fundamental research grant scheme under vote number 79346 .

Edited by: D. Solomatine

\section{References}

Adamowski, J. and Sun, K.: Development of a coupled wavelet transform and neural network method for flow forecasting of non-perennial rivers in semi-arid watersheds, J. Hydrol., 390, 85-91, 2010.

Affandi, A. K. and Watanabe, K.: Daily groundwater level fluctuation forecasting using soft computing technique, Nat. Sci., 5, 1-10, 2007.

Aqil, M., Kita, K., and Macalino, M.: A Preliminary study on the suitability of data driven approach for continuous water laeve modeling, Int. J. Comp. Sci., 1, 246-252, 2006.

Asefa, T., Kemblowski, M., McKee, M., and Khalil, A.: Multi-time scale stream flow predictions: The support vector machines approach, J. Hydrol., 318, 7-16, 2006.

Behzad, M., Asghari, K., Eazi, M., and Palhang, M.: Generalization performance of support vector machines and neural networks in runoff modeling, Expert Syst. Appl., 36, 7624-7629, 2009.

Birkinshaw, S. J., Parkin, G., and Rao. Z.: A hybrid neural networks and numerical models approach for predicting groundwater abstraction impacts, J. Hydroinform., 10, 127-137, 2008.

Box, G. E. P. and Jenkins, G.: Time Series Analysis, Forecasting and Control, Holden-Day, San Francisco, CA, 1970.

Budayan, C., Dikmen, I., and Birgonul, M. T.: Comparing the performance of traditional cluster analysis, self-organizing maps and fuzzy C-means method for strategic grouping, Expert Syst. Appl., 36, 11772-11781, 2009.

Bowden, G. J., Dandy, G. C., and Maier, H. R.: Input determination for neural network models in water resources application. Part 1background and methodology, J. Hydrology, 301, 75-92, 2005.

Cao, L.: Support vector machines experts for time series forecasting, Neurocomputing, 51, 321-339, 2003.

Chang, F. J., Chang, L. C., and Wang, Y. S.: Enforced selforganizing map neural networks for river flood forecasting, Hydrol. Process., 21, 741-749, 2007.

Chang, P. C. and Liao, T. W.: Combining SOM and fuzzy rule base for flow time prediction in semiconductor manufacturing factory, Appl. Soft Comput., 6, 198-206, 2006.

Chang, P. C., Fan, C. Y., and Wang, Y. W.: Evolving CBR and data segmentation by SOM for flow time prediction in semiconductor manufacturing factory, J. Intell. Manuf., 20, 421-429, 2008.

Chen, K. Y. and Wang, C. H.: A hybrid SARIMA and support vector machines in forecasting the production values of machinery industry in Taiwan, Expert System Application, 32, 254-264, 2007.

Chen, S. K., Mangimeli, P., and West, D.: The comparative ability of Self-organizing neural networks to define cluster structure, Omega, International Journal of Management Science, 23, 271279, 1995. 
Cheung, K. H., Szeto, K. Y., and Tam, K. Y.: Maximum-entropy approach to identify time-series lag structure for developing intelligent forecasting systems, International Journal of Computational Intellegence and Organization, 1, 94-106, 1996.

Corzo, G. A. and Solomantine, D. P.: Baseflow separation techniques for modular artificial neural network modelling in flow forecasting, Hydrolog. Sci. J., 52, 491-507, 2007.

Corzo, G. A., Solomatine, D. P., Hidayat, de Wit, M., Werner, M., Uhlenbrook, S., and Price, R. K.: Combining semi-distributed process-based and data-driven models in flow simulation: a case study of the Meuse river basin, Hydrol. Earth Syst. Sci., 13, 1619-1634, doi:10.5194/hess-13-1619-2009, 2009.

Douglas, E. M., Vogel, R. M., and Kroll, C. N.: Trends in floods and low flows in the United States: impact of spatial correlation, J. Hydrol., 240, 90-105, 2000.

Dibike, Y. B., Velickov, S., Solomatine, D. P., and Abbott, M. B.: Model induction with support vector machines: introduction and applications, ASCE J. Comput. Civil Eng., 15, 208-216, 2001.

Dibike, Y. D. and Solomatine, D. P.: River flow forecasting using artificial neural networks, Phys. Chem. Earth (B), 26, 1-7, 2001.

Dolling, O. R. and Varas, E. A.: Artificial neural networks for streamflow prediction, J. Hydraul. Res., 40, 547-554, 2003.

Elshorbagy, A., Corzo, G., Srinivasulu, S., and Solomatine, D. P.: Experimental investigation of the predictive capabilities of data driven modeling techniques in hydrology - Part 1: Concepts and methodology, Hydrol. Earth Syst. Sci., 14, 1931-1941, doi:10.5194/hess-14-1931-2010, 2010a.

Elshorbagy, A., Corzo, G., Srinivasulu, S., and Solomatine, D. P.: Experimental investigation of the predictive capabilities of data driven modeling techniques in hydrology - Part 2: Application, Hydrol. Earth Syst. Sci., 14, 1943-1961, doi:10.5194/hess-141943-2010, 2010b.

Fan, S. and Chen, L.: Short-term load forecasting based on an adaptive hybrid method, IEEE Transactions on Power System, 21, 392-401, 2006.

Fan, S., Mao, C., and Chen, L.: Next-day electricity-price forecasting using a hybrid network. IET. Gener. Transm. Distrib. 1, 176182, 2007.

Fernandez, C. and Vega, J. A.: Streamflow drought time series forecasting: a case study in a small watershed in north west spain, Stoch. Environ. Res. Risk Assess., 23, 1063-1070, 2009.

Firat, M.: Comparison of Artificial Intelligence Techniques for river flow forecasting, Hydrol. Earth Syst. Sci., 12, 123-139, doi:10.5194/hess-12-123-2008, 2008.

Firat, M. and Gungor, M.: Hydrological time-series modeling using an adaptive neuro-fuzzy inference system, Hydrol. Process., 18, 833-844, 2007.

Gencoglu, M. T. and Uyar, M.: Prediction of flashover voltage of insulators using least square support vector machines, Expert Systems with Applications, 36, 10789-10798, 2009.

Grayson, R. B., Moore, I. D., and McMahon, T. A.: Physically based hydrologic modelling. 2. Is the concept realistic, Water Resour. Res., 28, 2659-2666, 1992.

Goswami, M., O'Connor, K. M., Bhattarai, K. P., and Shamseldin, A. Y.: Assessing the performance of eight real-time updating models and procedures for the Brosna River, Hydrol. Earth Syst. Sci., 9, 394-411, doi:10.5194/hess-9-394-2005, 2005.
Hamilton, J. P., Whitelaw, G. S., and Fenech, A.: Mean annual temperature and annual precipitation trends at Canadian biosphere reserves, Envir. Monit. Assess, 67, 239-275, 2001.

Hanbay, D.: An expert system based on least square support vector machines for diagnosis of valvular heart disease, Expert Systems with Applictions, 36, 8368-8374, 2009.

Hecht-Nielsen, R.: Neurocomputing, Menlo Park, CA., AddisonWesley, 1990

Herbst, M. and Casper, M. C.: Towards model evaluation and identification using Self-Organizing Maps, Hydrol. Earth Syst. Sci., 12, 657-667, doi:10.5194/hess-12-657-2008, 2008.

Herbst, M., Gupta, H. V., and Casper, M. C.: Mapping model behaviour using Self-Organizing Maps, Hydrol. Earth Syst. Sci., 13, 395-409, doi:10.5194/hess-13-395-2009, 2009.

Hsu, K., Gupta, H. V., Gao, X. Sorooshian, S., and Imam, B.: Selforganizing linear output map (SOLO): an artificial neural network suitable for hydrologic modeling and analysis, Water Resour. Res., 38, 1302, doi:10.1029/2001WR000795, 2002.

Hsu, S.-H., Hsieh, J. J. P.-A., Chih, T.-C., and Hsu, K.-C.: A twostage architecture for stock price forecasting by integrating selforganizing map and support vector regression, Expert Systems with Applications, 36, 7947-7951, 2009.

Huang, C. L. and Tsai, C. Y.: A hybrid SOFM-SVR with a filterbased feature selection for stock market forecasting, Expert Syst. Appl., 36, 1529-1539, 2009.

Huang, W., Bing Xu, B., and Hilton, A.: Forecasting flow in apalachicola river using neural networks, Hydrol. Process., 18 , 2545-2564, 2004.

Hung, N. Q., Babel, M. S., Weesakul, S., and Tripathi, N. K.: An artificial neural network model for rainfall forecasting in Bangkok, Thailand, Hydrol. Earth Syst. Sci., 13, 1413-1425, doi:10.5194/hess-13-1413-2009, 2009.

Jain, A. and Kumar, A. M.: Hybrid neural network models for hydrologic time series forecasting, Appl. Soft Comput, 7, 585-592, 2007.

Jacobs, R. A., Jordon, M. A., Nowlan, S. J., and Hinton, G. E.: Adaptive mixtures of local expert, Neural Compt., 3, 79-87, 1991.

Juhos, I., Makra, L., and Toth, B.: Forecasting of traffic orgin NO and $\mathrm{NO}_{2}$ concentrations by Support Vector Machines and neural networks using principal component analysis, Simulation Modeling Practice and Theory, 16, 1488-1502, 2008.

Kang, S.: An investigation of the Use of Feedforward Neural Network for Forecasting, Kent State University, Ph.D. Thesis, 1991.

Kang, Y. W., Li, J., Cao, G. Y., Tu, H. Y., Li, J., and Yang, J.: Dynamic temperature modeling of an SOFC using least square support vector machines, J. Power Sources, 179, 683-692, 2008.

Kalteh, A. M., Hjorth, P., and Berndtsson, R.: Review of the selforganizing map (SOM) approach in water resources: Analysis, modeliong and application. Environ. Model. Softw., 23, 835845, 2008.

Khashei, M. and Bijari, M.: An artificial neural network (p,d,q) model for time series forecasting, Expert Syst. Appl., 37, 479489, 2010 .

Keskin, M. E. and Taylan, D.: Artifical models for interbasin flow prediction in southern Turkey, 14, 752-758, 2009.

Kisi, O.: River flow modeling using artificial neural networks, J. Hydrol. Eng., 9, 60-63, 2004. 
Kisi, O.: River flow forecasting and estimation using different artificial neural network technique, Hydrol. Res., 39.1, 27-40, 2008.

Kisi, O. and Cimen, M.: A wavelet-support vector machine conjunction model for monthly streamflow forecasting, J. Hydrol., 399, 132-140, 2010.

Kohonen, T.: Self-Organizing Maps, New York,Springer, 501, 2001.

Legates, D. R. and McCabe Jr., G. J.: Evaluating the use of goodness-of-fit measures in hydrologic and hydroclimatic model validation, Water Resour. Res., 35, 233-241, 1999.

Lin, G. F. and Chen, L. H.: Time series forecasting by combining the radial basis function network and the Self-Organizing Map, Hydrol. Process., 19, 1925-1937, 2005.

Lin, G. F. and Chen, L. H.: Identification of homogeneous regions for regional frequency analysis using the Self-Organizing Map, J. Hydrol., 324, 1-9, 2006.

Lin, G. F. and Wu, M. C.: A SOM-based approach to estimating design hyetographs of ungauged sites, J. Hydrol., 339, 216-226, 2007.

Lin, G. F. and Wu, M. C.: A hybrid neural network model for typhoon-rainfall forecasting, J. Hydrol., 375, 216-226, 2009.

Lin, J. Y., Cheng, C. T., and Chau, K. W.: Using support vector machines for long-term discharge prediction, Hydrol. Sci. J., 51, 599-612, 2006.

Liu, L. and Wang, W.: Exchange rates forecasting with least squares support vector machines, International Conference on Computer Science and Software Engineering, 1017-1019, 2008.

Luchetta, A. and Manetti, S.: A real time hydrological forecasting system using a fuzzy clustering approach, Comput. Geosci., 29, 1111-1117, 2003.

Luk, K. C., Ball, J. E., and Sharma A.: A study of optimal model lag and spatial inputs to artificial neural network for rainfall forecasting, J. Hydrol., 227, 56-65, 2000.

Maier, H. R. and Dandy, G. C.: Neural networks for the production and forecasting of water resource variables: a review and modelling issues and application, Environ. Model. Softw., 15, 101124,2000

Mangiameli, P., Chen, S. K., and West, D.: A comparison of SOM neural network and hierarchical clustering methods, Eur. J. Oper. Res., 93, 402-417, 1996.

Modarres, R.: Streamflow drought time series forecasting, Stoch. Environ. Res. Risk Assess., 21, 223-233, 2007.

Moreno, D., Marco, P., and Olmeda, I.: Self-Organizing Maps could improve the classification of Spanish mutual funds, Eur. J. Oper. Res., 147, 1039-1054, 2006.

Muhamad, J. R. and Hassan, J. N.: Khabur River flow using artificial neural networks, Al-Rafidain Engineering, 13, 33-42, 2005.

Murao, H., Nishikawa, I., Kitamura, S., Yamada, M., and Xie, P.: A hybrid neural network system for the rainfall estiamtion using saellite imagery. In: Proceedings of International Joint Conference on Neural Networks, IEEE press, 1211-1214, 1993.

Pai, P. F. and Lin, C. S.: A hybrid ARIMA and support vector machines model in stock price forecasting, Omega, 33, 497-505, 2005.

Pal, N. R., Pal, S., Das, J., and Majumdar, K,: SOFM-MLP: A hybrid neural network for atmospheric temperature prediction, IEEE T. Geosci. Remote Sens., 41, 2783-2791, 2003.

Partal, T. and Kisi, O.: Wavelet and neuro-fuzzy conjuction model for precipitation forecasting, J. Hydrol., 342, 199-212, 2007.
Refenes, A., Burgess, A. N., and Bents, Y.: Neural networks in financial engineering: A study in methodology, IEEE T. Neural Networ., 8, 1223-1267, 2003.

Roadknight, C. M., Balls, G. R., Mills, G.E. and Palmer-Brown, D. Modeling complex environmental data, IEEE T. Neural Networ., 8, 852-862, 1997.

Rui Xu, D. C. W.: Clustering, IEEE, 64-142, 2009.

Sharda, R. and Patil, R. B.: Connectionist approach to time series prediction: an empirical test, J. Intell. Manuf., 3, 317-323, 1992.

Sivapragasam, C. and Liong, S. Y.: Flow categorozation model for improving forecasting, Nord. Hydrol., 36, 37-48, 2005.

Solomatine, P. D., Maskey, M., and Shrestha, D. L.: Instance-based learning compared to other data-driven methods in hydrological forecasting, Hydrol. Process., 22, 275-287, 2008.

Srikanthan, R. and McMahon, T. A.: Stochastic generation of annual, monthly and daily climate data: A review, Hydrol. Earth Syst. Sci., 5, 653-670, doi:10.5194/hess-5-653-2001, 2001.

Srinivas, V. V., Tripathi, S., Rao, A. R., and Govindaraju, R. S.: Regional flood frequency analysis by combining self-organizing feature map and fuzzy clustering, J. Hydrol., 348, 148-166, 2009.

Suykens, J. A. K., Van Gestel, T., De Brabanter, J., De Moor, B., and Vandewalle, J.: Least squares support vector machines, World Scientific, Singapore, 2002.

Tang, Z. and Fishwick, P. A.: Feedforward Neural Nets as Models for Time Series Forecasting, ORSA Journal on Computing, 5, 374-385, 1993.

Tay, F. E. H. and Cao, L. J.: Improved financial time series forecasting by combining support vector machine with self- organizing feature map, Intell. Data Anal., 5, 339-354, 2001.

Vapnik, V.: The nature of Statistical Learning Theory, Springer Verlag, Berlin, 1995.

Vesanto, J. and Alhoniemi, E.: Clustering of the self-organizing map, IEEE T. Neural Networ., 11, 586-600, 2000.

Viessman, W. J., Lewis, G. L., and Knapp, J. W.: Introduction To Hydrology, Singapore, Harper \& Row, 1989.

Wang, H. and Hu D.: Comparison of SVM and LS-SVM for Regression, IEEE, 1, 279-283, 2005.

Wang, J. and Yan, D.: A high precision prediction method by using combination of ELMAN and SOM neural networks. In Proceeding of the first international symposium on neural networks China, 943-949, 2004.

Wang, W. C., Chau, K. W., Cheng, C. T., and Qiu, L.: A Comparison of Performance of Several Artificial Intelligence Methods for Forecasting Monthly Discharge Time Series, J. Hydrol., 374, 294-306, 2009.

Wang, W., Pieter H. A. J. M. V. G., Vrijling, J. K., and Ma, J.: Forecasting daily streamflow using hybrid ANN models, J. Hydrol., 324, 383-399, 2006.

Wong, F. S.: Time series forecasting using backpropagation neural networks, Neurocomputing, 2, 147-159, 1991.

Wu, C. L. and Chau, K. W.: A hybrid model coupled with singular spectrum analysis for daily rainfall prediction, J. Hydroinform., 12.4, 458-473, 2010.

Wu, C. L., Chau, K. W., and Li, Y. S.: Predicting monthly streamflow using data-driven models coupled with datapreprocessing techniques, Water Resour. Res., 45, W08432, doi:10.1029/2007wr006737, 2009. 
Wu, S. and Chow, T. W. S.: Clustering of the self-organizing map using a clustering validity index based on inter-cluster and intracluster density, Pattern Recognition, 37, 175-188, 2004.

Yu, P. S., Chen, S. T., and Chang, I. F.: Support vector regression for real-time flood stage forecasting, J. Hydrol., 328, 704-716, 2006.

Yue, S. and Wang, C.: The Mann-Kendall Test Modified by Effective Sample Size to Detect Trend in Serially Correlated Hydrological Series, Water Resour. Manage., 18 , 201-218, 2004.

Yunrong, X. and Liangzhong, J.: Water quality prediction using LS-SVM with particle swarm optimization, Second International Workshop on Knowledge Discovery and Data Mining, 900-904, 2009.
Zhang, B. and Govindaraju, G.: Prediction of watershed runoff using Bayesian concepts and modular neural network, Water Research Res., 36, 752-762, 2000.

Zhang, G., Patuwo, B. E., and Hu, M. Y.: Forecasting with artificial neural networks: the state of the art, Int. J. Forecast., 14, 35-62, 1998.

Zhang, G. P.: Time series forecasting using a hybrid ARIMA and neural network model, Neurocomputing, 50, 159-175, 2003.

Zou, H. F., Xia, G. P., Yang, F. T., and Wang, H. Y.: An investigation and comparison of artificial neural network and time series models for chinese food grain price forecasting, Neurocomputing, 70, 2913-2923, 2007. 